\title{
Power Fairness for Multicast Traffic in Optical Networks with Adaptive Light Splitters
}

\author{
Shadi Jawhar, Bernard Cousin, Zeina Bitar \\ IRISA laboratory, University of Rennes 1, Campus universitaire de Beaulieu, 35042, France \\ Bernard.Cousin@irisa.fr
}

\begin{abstract}
To perform data multicasting in the optical layer, optical nodes must be equipped with light splitters. Light splitters can split one light wave to more than one node output. A lot of work had been done in order to enhance the structure of the light splitter in a way to reduce its cost and enhance its performance in terms of the power loss resulted of multiple splitting. To guarantee the fairness of power received by different members of a same multicast group, the use of adaptive light splitters is required. Adaptive light splitters allow splitting an incoming light signal into two or more node output with the ability of varying the individual power of each output signal. This paper studies the benefits of using adaptive splitters on the value of the power received by each of the multicast group members in a way to assure fairness among all members.
\end{abstract}

\section{INTRODUCTION}

Fiber optics show a big evolution in terms of link capacity and speed compared to any other type of cables. This leads to more deployment of optical networks in the core of the international communication network, where wavelength division multiplexing assures efficient data transmission in the optical physical layer.

On the other side, multicasting had matured in the past years. This is because of the reduction in traffic which results when multiple clients requested to receive the same information from the same source. Since the basic mechanism of communication in a wavelength routed network is a light path, light trees must be generated to route data in the optical layer to all the group members [1]. A light path is an all-optical communication channel between two nodes in the optical network.

A light tree can be seen as a point-to-multipoint extension of a light path in which a group of light paths can deliver data to group members assigned to same multicast group source. To support multicasting in optical networks, optical nodes have to branch one incoming light wave to more than one output node port.
To be able to work as branching, optical nodes must be equipped with light splitters that split one light wave to more than one output.

Splitter sharing and adaptive splitting can enhance the overall network performance, to produce a reduction in the cost of the network deployment phase. Ordinary splitters proposed usually divide the light wave power equally between all the output signals [2]. Performances can be enhanced if adaptive splitters are used and the division of the output power is based on the distribution of the multicast group members.

This paper studies the benefits of using adaptive splitters and dynamically modifying their splitting output ratios in a way to enhance the power received by all the group members. Power received by group members is evaluated based on the criteria of fairness of power received at all destinations of the multicast group, which enhance the minimum received signal to noise ratio under a total power constraint.

This paper is organized as follows: in Section II, a description of the architecture of an ordinary light splitter is presented. In Section III, a full description of adaptive light splitters is given as described in main related works. In Section IV, an algorithm on how configuring the output power distribution of the adaptive splitters is done based on the group members distribution. In Section V, a performance evaluation between using ordinary light splitters and using of adaptive splitting is done. Results are then analyzed to show the enhanced efficiency when using those adaptive splitters.

\section{MULTICAST CAPABLE OPTICAL CROSS CONNECT USING ORDINARY LIGHT SPLITTERS}

An Optical Cross Connects OXCs is designed to switch an optical signal from an input port to an output port. For it to split one optical signal to multiple output ports, it must be equipped with an optical light splitter. 
Enhanced ordinary splitters can be configured to split an input signal into $\mathrm{m}$ outputs, $1<\mathrm{m}<\mathrm{P}$ (If $\mathrm{m}=1$, then there is no splitting. If $\mathrm{m}=\mathrm{P}$, then it is a broadcast splitting). Further configuration can switch each of the $m$ resulting signals to any desired output.

To do so, a Splitter-and-Delivery SaD [2] switch can be used. A $\mathrm{P} \times \mathrm{P} \mathrm{SaD}$ switch consists of $\mathrm{P}$ power splitters, $\mathrm{P} \times \mathrm{P}$ optical gates, and $\mathrm{P} \times \mathrm{P}$ photonic switching elements. It is designed in this way in order to reduce its cost. This structure also improves the power efficiency, which leads to a better received signal and less number of amplifiers to be used.

Multiple propositions for generating multicast trees on optical networks in which not all nodes have light splitters have been presented in [3][4] and [5]. Some of these proposals are based on additional signaling performed during or before data forwarding to generate trees, taking into consideration splitters location.

Others use re-route-to-source asking the source to send a second stream of data to be able to do multicasting in the optical layers. All the mentioned propositions assume that light splitters are only capable of splitting the input signal to multiple outputs with the same ratio.

\section{ADAPTIVE LIGHT SPLITTERS}

Adaptive light splitters are capable of doing advanced splitting mechanisms. One optical signal received at one input port of a node can be split and coupled into many output ports of the node with arbitrary splitting ratios.

For instance, an adaptive optical splitter structure employing an opto-VLSI processor and 4-f imaging system was proposed in [6]. The opto-VSLI processor comprises an array of liquid crystal (LC) cells which is capable of achieving arbitrary beam of deflection or multicasting. This technique allows to electronically controlling the input signal and how it is split into multiple outputs.

While normal splitter can only split an input power $\mathrm{P}$ to equal parts, where each part is $=\mathrm{P} / \mathrm{m}$ ( $\mathrm{m}$ is the number of output branches, $1<\mathrm{m}<\mathrm{n}, \mathrm{n}=$ total number of outputs), an adaptive splitter can split it into different ratios.

The structure of the proposed adaptive optical power splitter is shown in Figure 1. It consists of the opto-VLSI processor, a converging lens, and an optical fiber collimator array, aligned to form a 4-f imaging system.

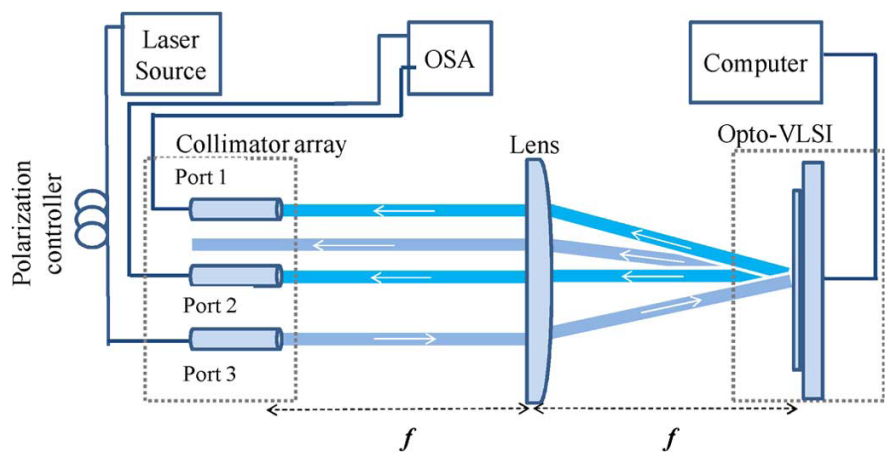

Figure 1 Adaptive optical splitter using an opto-VLSI processor and a 4-f imaging systems.

\section{ALGORITHM FOR CONTROLLING ADAPTIVE LIGHT SPLITTERS BASED ON GROUP MEMBERS DISTRIBUTION}

The section describes an algorithm to compute the splitting factors on the branching nodes equipped with adaptive light splitters, in a multicast tree to enhance both the average and the minimum power received by group members. This assures more fairness of the signal power received by group members.

As a result, uniformly distributing amplifiers will lead to a reduction in their number. Thus, fewer amplifiers would be needed and a better distribution of those amplifiers can be made.

We study the effect of splitting factor on both the cost of the generated trees which affects the power loss of signals received by group members.

The algorithm is based on modifying the splitting ratios in the adaptive splitters based on the number of downstream members on each of the outgoing output of the splitter.

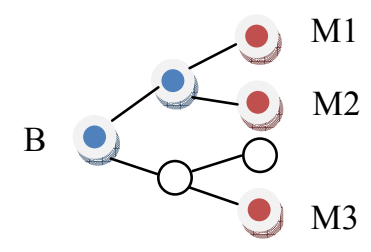

Figure 2 - Splitting ratio based on the number of splitters

The example shown in Figure 2 illustrates an example of a case where the branching node $\mathrm{B}$, splits the signal into two outputs. In the first output, two group members will receive the signal.

However, the second output of node $\mathrm{B}$ will deliver data to only one group member M3. As a result, a second branching will happen in the path of the first output because of the existence of the two group members M1 and M2. 
Assuming that the two branching nodes in this example are equally splitting the signal into each outputs, then the three members will receive different ratios of the power of the input signal. The two members $\mathrm{M} 1$ and $\mathrm{M} 2$ will each receive $1 / 4$ of the input signal power. However M3 will receives $1 / 2$ of the input signal power.

An attentive reader has already deduces the status of this bad solution: either the source of the signal must emit higher power to enable M1 or M2 to receive the signal with sufficient strength, or amplifiers must be configured and used.

If the source signal power is just sufficient to reach M3 thus the power signal strength will not be sufficient to be decoded by M1 or M2.

If the adaptive power capability of the splitter on the branching node $\mathrm{B}$ is well managed, then each of the three members will receive equal signal power.

Since the number of members on the first B output is double that on the second, and then signal power ratio must be twice on the first output.

As a result, the branching node $\mathrm{B}$ must split the signal into two different power ratios; the first is $2 / 3$ of the power input, while the second is $1 / 3$ of the power input. In this way, each of the three members $\mathrm{M} 1, \mathrm{M} 2, \mathrm{M} 3$ will receive $1 / 3$ of the input signal power.

In order to assure that all the group members receive about the same ratio of the signal power issued from the source, the branching ratios on all the branching nodes of a multicast tree must be performed based on the number of group members spanning downstream on each of the outputs of the splitters.

As a result, some exchange of information must be done between the group members and the branching nodes. This exchange must inform each of the branching nodes with how many members are found on each of the branching outputs.

A branching node with an adaptive light splitter, must know how many group members are available on each of its output branches. This way the branching node can split the signal power based on these numbers. The splitting power ratio on each output must be inversely proportional to the number of members on the output.

To achieve this, each group member of the multicast group must send a notification message upstream to the source.

This message may be received by a non-branching node, or a branching node, for the multicast group in consideration. When a non-branching node, receives the message, it will forward it upstream without any action.
When this message is received by one of the branching nodes for the considered multicast group, the branching node must save the message information about the number of group members, by associating a counter to each of its output branches.

After that, the branching node forwards the message with the total number of its group members upstream to the source, so it can be received by other possible branching nodes upstream.

When all group members have sent their notification messages, the counters on all the outputs of every branching node in the multicast tree in consideration are updated.

The example in Figure 3 shows how the notification messages are sent by group members and managed by optical nodes.

B

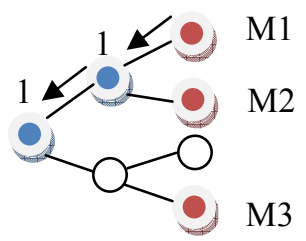

B

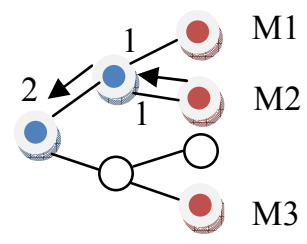

$\mathrm{B}$

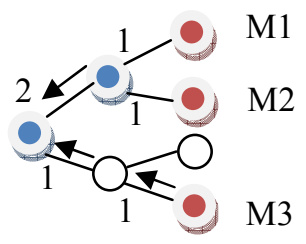

Figure 3 - Notification messages sent by the group members (in red) and managed by optical nodes (in blue)

After the exchange of those notification messages, each branching nodes will have complete information, in terms of the number of group members for any multicast data will be forwarded later.

The number of group members on each of the output branches, will be an important parameter in the adaptive splitting mechanism. Each of the adaptive light splitters will modify its splitting ratio for each of the outputs based on the updated information. It assures that group members will receive the same power ratios of the source signal. 


\section{Simulations AND RESUlts}

In order to demonstrate that benefits of the use of the adaptive splitting, performance evaluation is performed. We compare the effect of use of adaptive splitters versus the use of ordinary splitters.

Results are measured in terms of the power of signal received by each of the group members, which affects the signal to noise ratio in case amplifiers are used.

The network topology shown in figure 4 is made up of optical nodes interconnected by optical links. We consider the US IP backbone network as the realistic topology to study. It is a very well-known topology, used by many similar works.

We consider that all the links in the network are identical. We realize that nodes in this network are either connected to $2,3,4$ or maximum 5 other nodes.

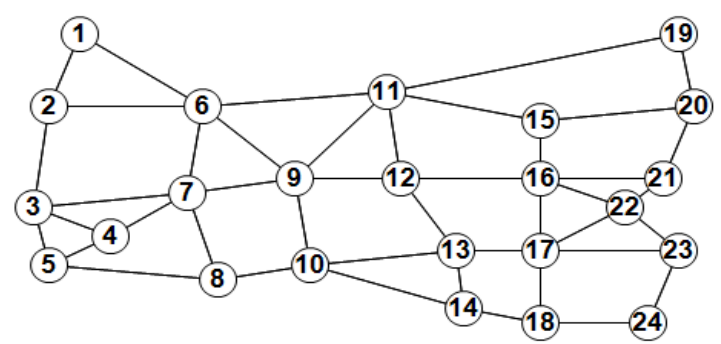

Figure 4 US IP backbone network

We assume that nodes in this network are either connected to 2,3 , and 4 . It is justified because the network topology is rather small and splitter rather expensive. Thus, splitters that need to be placed on this network can be $1 \times 2,1 \times 3$, or $1 \times 4$ types. We then distribute the splitters on nodes with highest degree because these nodes are more likely to do branching. It has been shown [7] that this distribution gives good results.

In the first scenario, we assume that all the splitters are ordinary light splitters which are chosen to have a splitting capability equal to 4 , corresponding to the highest node degree, which is 5 . Since splitting happens from one input to multiple outputs, then the maximum splitting capability required is 4 .

In the second scenario, we assume that all splitters are adaptive light splitters. As a result, each splitter configures its splitting ratios based on the number of group members spanning on each of the output branches.

We consider distributing 6 light splitters, thus $25 \%$ of the network nodes can be equipped by a splitter. The 6 splitters are placed on nodes $6,7,9,11,16$ and 17 . These nodes are the ones with highest number of neighbors [8] [9].

Figure 5 shows the 6 splitters distributed on the nodes with highest node degree.

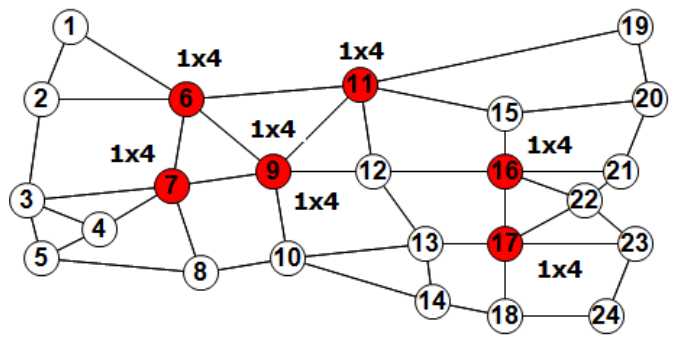

Figure 5 - Distributing 6 splitters (node in red) each with $1 \times 4$ splitting factor

We consider 24 × 20 random multicast groups; each time the source is placed on one different node. Group members are then randomly chosen among the network. We vary the size of the group in order to simulate different sizes of groups. For each group size, varying the source on all nodes of the network and randomly generating 20 member groups will produce significant simulation results.

Multicast trees are generated by applying the shortest path algorithm, from the source to the group members. Blocking can occur when a multicast node needs with no light splitter is requested to branch to more than one outgoing link. In this case, it locates an upstream node to do the branching and generate the associated multicast tree [10]. In paper [10], multicasting under light splitters constraints is treated to generate the trees in an efficient way, and with the lower cost in terms of used links. Splitting factor capability configured in an $\mathrm{SaD}$ component has an important influence on the power of each of the branched signals.

When using ordinary light splitters, if a signal is branched to $m$ output signals, then each of the output signals power is $1 / \mathrm{m}$ of the input power. When using the adaptive light splitters, if a signal is branched to $m$ outputs, then each of the output signal power is equal to the number of group members spanning on the branch divided by the total number of group members over all branches.

Simulation is done to compare the power received by group members in each of the two scenarios. Simulation shows that the average, and the minimum power received by group members is higher in case when adaptive light splitters are used.

In order to measure the network design cost, the number of amplifiers placed, their gain, and their locations play an important role. Quality and quantity of amplifiers is defined by the power loss caused by transmission and splitting. To measure the power loss on different nodes of the tree, the maximum optical power loss ratio, MXOPLR is defined. This term measure the splitting power loss at all nodes, and returns the ratio by which the node that received the least power is fractioned. 
Suppose that at each splitter, a factor is defined to measure the input power compared to the output power. If a node receives messages after successive splittings, then the ratio of power is the result of the product of multiple splitting factors that occur on the branch from the source to the node.

MXOPLR(T) is the maximum power ratio on all the branches of the generated tree $\mathrm{T}$. The MXOPLR criteria is given by the formula below, where $\mathrm{SF}_{\mathrm{i}}$ is the splitting factor of each Split-and-Delivery switch $i$ used at each light splitter on the tree $\mathrm{T}(\mathrm{SaD}(\mathrm{T}))$.

$$
\operatorname{MXOPLR}(\mathrm{T})=\operatorname{Max}\left(\prod_{\substack{i \in \operatorname{SaD}(T) \\ S F i>1}} S F_{i}\right)^{\prime}
$$

The MXOPLR criteria measure the power level of the signal with the least power received by any of the group members. This in fact gives an indication of the member that receives a signal with a very low power due to successive splitting with high splitting factors.

As shown in figure 6, the maximum power loss ratio is lower with adaptive splitters than with ordinary splitters. Adaptive splitters that splits the input signal into controlled rations, assures that all the group members will receive the same ratio of the input power. However, ordinary splitters gives no attention to the number of group members spanning on each of its output branches, as it only recognizes on which branches it must do the splitting.

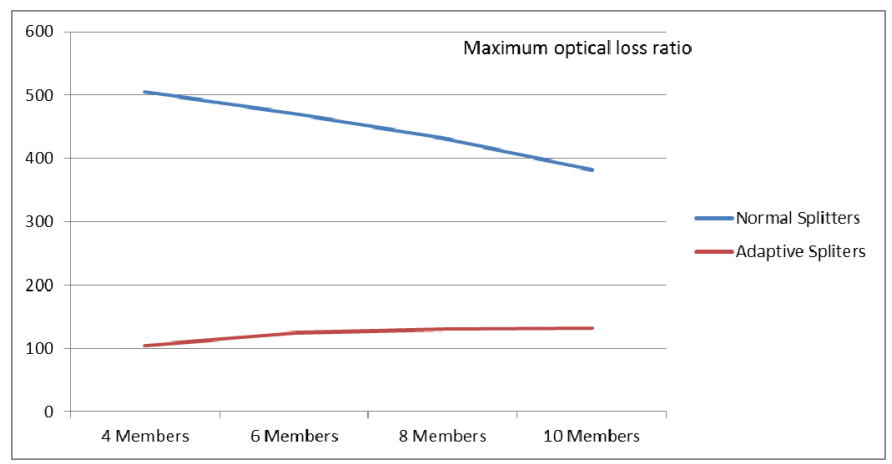

Figure 6 -Maximum optical power loss ratio

The next table shows the MXOPLR and the number of splitters used on branching nodes in each of the two cases. This will gives a good idea on the minimum power received by group members, which in turns, interprets the $\mathrm{S} / \mathrm{N}$ ratio.

\begin{tabular}{|c|c|c|}
\hline Group Size & Ordinary Splitters & Adaptive Splitters \\
\hline 4 & $506 / 64$ & $105 / 64$ \\
\hline 6 & $471 / 71$ & $125 / 71$ \\
\hline 8 & $432 / 78$ & $130 / 78$ \\
\hline 10 & $392 / 72$ & $131 / 72$ \\
\hline
\end{tabular}

The numbers in the table above shows clearly that when ordinary splitters are used, the chance to have a group member that receives a signal with power very low compared to other members is higher.

\section{CONCLUSION}

Recently adaptive splitting capability has been added to optical splitters. It adds the ability to dynamically split the input signal into different power ratios on the output branches of an optical switch. This enables the splitter to control the quantity of power given to each of the outputs.

To benefit of this added feature in multicasting in optical networks, the splitter must distribute the power based on the distribution of the group members. Thus, each splitter must know the number of members spanning from each of its output branches. We have proposed a sketchy algorithm to achieve this knowledge. This quite simple algorithm could be merged into a multicast tree algorithm, thus it will come at no additional cost.

We reserved for another study the protocol implementation and the evaluation of its message cost. The above knowledge will assure that all the group members receive the same ratio of the signal power issued from the source. As a result, all group members will have fairness in the distribution in terms of the power they receive.

Obviously the advantage of our adaptive splitting solution comes at a cost. Currently at less, the cost of an adaptive splitter is higher than the cost of an ordinary splitter. The real additional cost remains to be evaluated. However we could hope that this additional cost will be drastically reduced if adaptive splitter solutions are widely deployed.

\section{REFERENCES}

[1] Marcos Rogério Salvador, Sonia M. Heemstra de Groot, Diptish Dey. "An All-Optical WDM Packet-Switched Network Architecture with Support for Group Communication", IEEE International Conference on Networking, 2001.

[2] W. S. Hu, Q. J .Zeng. "Multicasting Optical Cross Connects Employing Splitter-And-Delivery Switch", IEEE Photonics Technology Letters, 1998.

[3] Fen Zhou, Miklos Molnar, Bernard Cousin, Gwendal Simon. "Power-optimal Design of Multicast Light-trees in WDM Networks". IEEE Communications Letters. Volume 15, Issue $\mathrm{n}^{\circ}$ 11, Page(s): 1240 - 1242. November 2011

[4] M. Ali and J. Deogun. "Power-efficient Design of Multicast Wavelength Routed Networks," IEEE Journal on Selected Areas in Communications, 2000. 
[5]

G. N. Rouskas: "Light-tree Routing under Optical Layer Power Budget Constraints," IEEE Computer Commununications Workshop 2002.

[6] Haithem A. B. Mustafa, Feng Xiao, and Kamal Alameh. "Adaptive Optical Splitter Employing an Opto-VLSI Processor and a 4-f Imaging System". $7^{\text {th }}$ International Symposium on High Capacity Optical Networks and Enabling Technologies 2010.

[7] Oliver $\mathrm{Yu}$ and Yuan Ca. "Placement of Light Splitters and Wavelength Converters for Efficient Multicast in All-Optical WDM Networks", IEICE Transactions on Information and Systems, 2006.

[8] Shadi Jawhar, Bernard Cousin, Samer Lahoud. "Efficient Placement of Light Splitters in Heterogeneous Optical Networks," $7^{\text {th }}$ International Symposium on High Capacity Optical Networks and Enabling Technologies 2010.

[9] Maher Ali, Jitender Deogun. "Allocation of Splitting Nodes in All-Optical Wavelength-Routed Networks", Photonic Network Communications, 2000.

[10] Shadi Jawhar, Bernard Cousin. "Optical Multicast Routing Under Light Splitter Constraints," $7^{\text {th }}$ International Conference on Information Technology: New Generations, 2010.

[11] I. Chlamtac, A. Ganz, and G. Karmi, "Lightpath Communications: An Approach to High Bandwidth Optical WAN's," IEEE Transactions on Communications, 1992.

[12] Yufeng Xin, George N. Rouskas. "Multicast Routing Under Optical Layer Constraints", IEEE Infocom, 2004.

[13] W.-Y. Tseng, S.-Y Kuo. "All-optical Multicasting on Wavelength Routed WDM Networks with Partial Replication". IEEE, International Conference on Intelligence in Networks, 2001.

[14] Chunming Qiao, Myoungki Jeong, Amit Guha, Xijun Zhang, John Wei. "WDM Multicasting in IP over WDM Networks", Seventh Annual International Conference on Network Protocols, 1999.

[15] Jianping Wang, Xiangtong Qi, Biao Chen. "Wavelength Assignment for Multicast in All-optical WDM Networks with Splitting Constraints", IEEE/ACM Transactions on Networking, 2006.

[16] Towsif Mannan, BinWang. "Dynamic Multicast Session Provisioning in WDM Optical Networks with Sparse Splitting Capability", International Conference on Communications and Computer Networks, 2006.
[17] Shuguang Yan, Jitender Deogun, Maher Ali. "Routing in Sparse Splitting Optical Networks with Multicast Traffic", Computer Networks, 2002 